\title{
Adequacy Score for the availability of essential drugs of an institution
}

\author{
I.R.H.S. Ihalagama ${ }^{1 *}$, C.A.D.M.N.C. Kolambage ${ }^{1}$, T.U. Subasinghe ${ }^{1}$, W.D. Sudasinghe ${ }^{1}$ \\ 1 Post Graduate Institute of Medicine, University of Colombo, Sri Lanka \\ * sathyajithihalagama@gmail.com \\ https://orcid.org/0000-0002-0285-4355
}

\begin{abstract}
Introduction: Sri Lanka classifies a set of essential drugs for the country in their "National List of Essential Medicines". The drugs in the list are expected to be available in the functioning health systems at all times; in adequate amounts and in appropriate dosage forms, with assured minimum quality, at a price people can afford. However, there is hardly any mechanism to keep track of the adequacy of essential drugs. Hence, the scope of this study is to take a step forward from there, by defining an adequacy score for the adequacy of the essential drugs in a specific institution for a defined period.
\end{abstract}

Methods: The drug consumption, institutional admissions and out-patient visits for the institution were analyzed. The available models also were identified and studied. A model was developed to calculate available drug volume (adjusted to a time period) against a predicted volume of the drug. The predicted volume of drugs that should be in the stock was then calculated by using a neural network applying the previous data. The adequacy of each individual drug was calculated. The percentage of the mean of adequacy score for each drug was expressed as adequacy score for the list of essential medicine.

Results: This model has doubled the accuracy of a non-time adjusted model in predicting the adequacy of a drug as this includes the temporal distribution of the volume of drugs. It eliminates overestimation of the stocks at the point of reception of new stock and eliminates the underestimation in the pre-reception period. Each drug in the list was assigned a dynamic adequacy score.

Conclusions: The new model has improved the accuracy against the non-time adjusted model. The generated score is dynamic and updated along the time axis, therefore has the potential to be used as a performance indicator of the institution as well. The model can be scaled-up adding more data elements in the prediction of the needed volume of drugs. However, the model may need further validation based on the practically relevant independent parameters.

Keywords: Essential drugs, Drug Volume Prediction Model, Essential Drug Adequacy Score 